\title{
ON ARCHIMAX COPULAS AND MULTIVARIATE GPD MODELS
}

\section{BARRO DIAKARYA and SOUMAÏLA MOUSSA}

Université Ouaga II

12 BP: 417 Ouagadougou

Burkina Faso

e-mail: dbarro2@gmail.com

FST, Dépt de Maths et Info

Université AM

BP 10662 Niamey

Niger

\begin{abstract}
This paper develops an extension to three dimensional study some properties of Archimax copulas. Moreover, multivariate generalized Pareto distributions are characterized by a pseudo-dependence function while usual multivariate extremal models are given with the corresponding dependence function.
\end{abstract}

\section{Introduction}

The concept of Archimedean copula is inherently related to Archimedean generator. So, the class of Archimedean copulas form a parameterized by real-valued functions. An $n$-dimensional copula $C$ is one-dimensional generator functions (see [4]). Arising in the context of 
Laplace transform (Joe) Archimedean copulas form these last years an important family that allows multivariate dependence modelling involving one-dimensional generator functions (see [1]). An $n$-dimensional copula $C$ is an Archimedean copula if there exists a continuous, strictly decreasing and convex function $\varphi:[0,1] \rightarrow[0,+\infty]$, completely monotone on $[0 ; \infty]$, and which is $(n-2)$ times derivative and $(-1)^{k}\left(\varphi^{-1}\right)^{(k)}(t) \geq 0$ for all $k=1,2, \ldots, n-2$, called the Archimedean generator function of $C$, such as for all $\left(u_{1}, \ldots, u_{n}\right) \in[0,1]^{n}$,

$$
C\left(u_{1}, \ldots, u_{n}\right)=\varphi\left[\varphi^{-1}\left(u_{1}\right)+\ldots+\varphi^{-1}\left(u_{n}\right)\right],
$$

where the generalized inverse of $\varphi^{-1}(y)=\inf \{t \in[0,1]: \varphi(t) \leq y\}$ (see [6]).

Among the most usual properties of Archimedean copulas that they present analytic form given by the generator, their exchangeability and positivity and positive and quadrant dependence of the underlying bivariate random vectors (see [1]). However in multivariate analysis applied in portfolio management, for example, we need non-exchangeable models. That is why authors use Laplace transforms to derive more flexible extension of this family of copulas by nesting of generators allowing different degrees of positive dependence in bivariate margins. By denoting an $m$-dimensional construction defined interactively for all

$$
\begin{aligned}
& \left(u_{1}, \ldots, u_{m}\right) \in[0,1]^{m} \text { by } \\
& \qquad C_{\psi}\left(u_{1}, \ldots, u_{m}\right)=\psi_{0}\left[\psi_{0}^{-1}\left(u_{1}\right)+\psi_{1}^{-1}\left(C_{\Psi_{1}}\left(u_{1}, \ldots, u_{m}\right)\right)\right] .
\end{aligned}
$$

This generalisation leads to the class of nested Archimedean copulas, an asymmetric subfamily of this class of copulas. Nested Archimedean copulas allow different levels of dependence between the components of the underlying random vector.

The main contribution of this study is to construct a new class of Archimax copulas in a three-dimensional study. Multivariate Pareto models are characterized and particular distributions are given in both in three dimension and higher cases. 


\section{Three Dimensional Extension of Archimax Copulas}

A bivariate member of this class with generator $\varphi$ is given, for all $(u, v) \in[0,1]^{2}$ by

$$
C_{\varphi, A}(u, v)=\varphi^{-1}\left[\min \left(\varphi(0),(\varphi(u)+\varphi(v)) A\left(\frac{\varphi(u)}{\varphi(u)+\varphi(v)}\right)\right)\right]
$$

where $A$ is a Pickands ([8]) dependence function mapping the unit simplex (see [1])

$$
S_{n}=\left\{x \in \mathbb{R}^{n}, \sum_{i=1}^{n}\|x\|_{1}=1,\|x\|_{1}=\sum_{i=1}^{n} x_{i}\right\} \text { of } \mathbb{R}^{n},
$$

to $\left[\frac{1}{n}, 1\right]$ satisfying $\max (t ; 1-t) \leq A(t) \leq 1$ for all $t \in[0 ; 1]$.

The class of Archimax copulas contains a special subclass of all Archimedean copulas (when $A(t) \equiv 1$ see [7]) and all extreme values copulas, then $\varphi(t)=-\log (t)$. Particularly every extreme value model (copula or distribution) is associated to a dependence function $A_{C}$ proposed by Pickands, subject to some convex constraint and defined on $S_{n}$ (see [8]). Then, for all $\left(u_{1}, \ldots, u_{n}\right) \in[0,1]^{n}$,

$$
C\left(u_{1}, \ldots, u_{n}\right)=\exp \left\{\sum_{i=1}^{n} \tilde{u}_{i} A_{G}\left(\frac{\tilde{u}_{i}}{\sum_{i=1}^{n} \tilde{u}_{i}}, \ldots \frac{\tilde{u}_{n-1}}{\sum_{i=1}^{n} \tilde{u}_{i}}\right)\right\}
$$

where $\tilde{u}_{i}=\log u_{i} ; i=1, \ldots, n$ (see [3] and [8]).

The following lemma introduces the main result of this section:

Lemma 1. Let $A$ be the Pickands dependence function and $\phi$ be a Laplace transform. Then, the univarite function defined such as $F_{t}(x)=\phi^{-1}(x) A\left(t_{1}, t_{2}\right)$ with $x \in \mathbb{R} ; t_{i} \geq 0 \in[0,1]$, where $t=\left(t_{1}, t_{2}\right) \in S_{2}$ is the distribution function of a random variable $X_{i}$. 
Proof. Since the function $A$ is defined from the unit simplex to $[0,1]$ and $0 \leq \phi^{-1}(x) \leq 1$ for all $x \in \mathbb{R}$. Therefore, for all $x>0,0 \leq \psi(x) \leq 1$. Furthermore, one have $\lim _{x \rightarrow 0^{+}} \psi(x)=0$ and $\lim _{x \rightarrow+\infty} \psi(x)=1$.

Moreover, for all $x \in \mathbb{R}$,

$$
\psi^{\prime}(x)=\left(\phi^{-1}\right)^{\prime}(x) A\left(t_{1}, t_{2}\right) \geq 0 .
$$

So $\psi$ is increasing and continuous. Finally, there exists a random variable $X$ such that $\psi(x)=P(X \leq x) \cdot \psi^{\prime}(x)$.

The following result gives a trivariate extension of Archimax copulas:

Proposition 2. For all bivariate Pickands dependence function A, i.e., satisfying (3), the parametric function defined, for all $\left(u_{1}, u, u_{3}\right) \in[0,1]^{3}$ by

$$
C\left(u_{1}, u, u_{3}\right)=\phi^{-1}\left[\left(\sum_{i=1}^{3} \phi^{-1}\left(u_{i}\right)\right) A\left(\frac{\phi^{-1}\left(u_{1}\right)}{\sum_{i=1}^{m} \phi^{-1}\left(u_{i}\right)}, \frac{\phi^{-1}\left(u_{2}\right)}{\sum_{i=1}^{m} \phi^{-1}\left(u_{i}\right)}\right)\right]
$$

is also a copula.

Proof. A sufficient condition for proving Proposition 2 is to build a continuous random vector $\left(X_{1}, X_{2}, X_{3}\right)$ associated to copula $C$. For this end, let $Y$ denote the univariate random with distribution with Laplace transform $\psi$, that is, $\psi(x)=\int_{0}^{+\infty} e^{-x t} d \psi(t)$.

Then, conditionally to $Y$ let $X=\left(X_{1}, X_{2}, X_{3}\right)$ be three independent random variables. It follows that

$$
\begin{aligned}
P\left(X_{1} \leq x_{1}, X_{2} \leq x_{2}, X_{3} \leq x_{3}\right) & =E\left[P\left(X_{1} \leq x_{1}, X_{2} \leq x_{2}, X_{3} \leq x_{3} / Y\right)\right] \\
& =E\left[\prod_{i=1}^{3} e^{-Y_{\varphi}\left(x_{i}\right)}\right] .
\end{aligned}
$$


Then, we obtain

$$
P\left(X_{1} \leq x_{1}, X_{2} \leq x_{2}, X_{3} \leq x_{3}\right)=\phi\left(\psi\left(x_{1}\right)+\psi\left(x_{2}\right)+\psi\left(x_{3}\right)\right),
$$

or

$$
P(X \leq x)=\phi\left[\sum_{i=1}^{3} \phi^{-1}\left(u_{i}\right) A\left(\frac{\phi^{-1}\left(u_{1}\right)}{\sum_{i=1}^{3} \phi^{-1}\left(u_{i}\right)}, \frac{\phi^{-1}\left(u_{1}\right)}{\sum_{i=1}^{3} \phi^{-1}\left(u_{i}\right)}\right)\right] .
$$

Furthermore, by definition,

$$
C_{\phi}\left(u_{1}, u, u_{3}\right)=P\left(X_{1} \leq H_{1}^{-1}\left(u_{1}\right), X_{1} \leq H_{1}^{-1}\left(u_{1}\right), X_{1} \leq H_{1}^{-1}\left(u_{1}\right)\right) .
$$

Then, the $H_{i}$ being the marginal distributions of $H$, by setting $H(x)=\phi(x)$, it comes that, for all $\left(u_{1}, u, u_{3}\right) \in[0,1]^{3}$;

$$
C_{\psi}\left(u_{1}, u, u_{3}\right)=\phi\left[\left(\sum_{i=1}^{3} \phi^{-1}\left(u_{i}\right)\right) A\left(\frac{\phi^{-1}\left(u_{1}\right)}{\sum_{i=1}^{m} \phi^{-1}\left(u_{i}\right)}, \frac{\phi^{-1}\left(u_{2}\right)}{\sum_{i=1}^{m} \phi^{-1}\left(u_{i}\right)}\right)\right] .
$$

So the relation (2.5) is obtained as asserted.

The following result is a consequence of direct consequence.

Corollary 3. For all Archimax triavariate copula $C_{A, \varphi}$ given by (2.3) with generator $\varphi$. Then, there exists a convex function $D_{A, \varphi}$ such as

$$
C\left(u_{1}, u, u_{3}\right)=\phi^{-1}\left[\left(\sum_{i=1}^{3} \phi^{-1}\left(u_{i}\right)\right) D_{A, \varphi}\left(\phi^{-1}\left(u_{1}\right), \phi^{-1}\left(u_{1}\right)\right)\right] .
$$

\section{A Characterization of Multivariate Generalized Pareto Models}

Let recall some properties on multivariate generalized Pareto distributions (GPD) via the corresponding multivariate extreme values (MEV). 
Definition 1 (see [5] and [10]). The multivariate generalized distribution of Pareto associated to a sample of random variables $X=\left(X_{1}, \ldots, X_{n}\right)$ :

$$
H(x)=1-\log \left(\frac{G(x)}{G(x \wedge 0)}\right)=\frac{-1}{\log G\left(x_{0}\right)} \log \left(\frac{G\left(x_{0}+x\right)}{G\left(x \wedge x_{0}\right)}\right) \text { if } x \geq 0
$$

with $x \wedge 0=\min (x, 0)$ and $x_{0} \in \operatorname{support}(G)$, where $G$ is the MEV distribution of the same.

The following result allows us to characterize MGP distributions. Consider Fréchet margin $Y_{i}=\frac{-1}{\log \left[F_{i}\left(X_{i}\right)\right]}$ of random variable $X_{i}$, that is, $Y_{i}$, i.e.,

$$
P\left[Y_{i} \leq y_{i}\right]=\exp \left(\frac{-1}{y_{i}}\right) ; y_{i}>0
$$

\subsection{The pseudo-dependence function of multivariate GPD}

Like the Pickands dependence function for the MEV distributions we propose convexe function which can characterize the family of GPD.

Proposition 2. Let $G$ be the multivariate generalized Pareto distribution associated to a sample of observations $X=\left(X_{1}, \ldots, X_{m}\right)$ then

$$
H(x)=1+\left\{-\sum_{i=1}^{m} y_{i}\left(x_{i}\right)\right\} B\left(\frac{y_{1}\left(x_{1}\right)}{\sum_{i=1}^{m} y_{i}\left(x_{i}\right)}, \ldots, \frac{y_{m-1}\left(x_{m-1}\right)}{\sum_{i=1}^{m} y_{i}\left(x_{i}\right)}\right),
$$

where $B$ is the pseudo-Pickands' dependence function of $H$, defined on the unit simplex $S_{m-1}=\mathbb{R}^{m-1}$. 
Proof. For a given $n \geq 1$, suppose $X_{n}=\left(X_{n 1}, \ldots, X_{n m}\right)$ are i.i.d random vectors with $m$-dimensional distribution $F$. Let

$$
M_{n}=\left(M_{n 1}, \ldots, M_{n m}\right)=\left(\max _{1 \leq i \leq n}\left(X_{i 1}\right), \ldots, \max _{1 \leq i \leq n}\left(X_{i m}\right)\right) .
$$

be the compentwise maxima of the set $\left\{X_{n}, n \geq 1\right\}$. A multivariate extreme value (MEV) distributions is a continuous and non degenerated function $G$, for which there exists normalising constants vectors:

$$
\sigma_{n}=\left(\sigma_{n 1}, \ldots, \sigma_{n m}\right) \text { with } \sigma_{n j}>0 \text { and } \mu_{n}=\left(\mu_{n 1}, \ldots, \mu_{n m}\right),
$$

with $\mu_{n j} \in \mathbb{R}$ for $j=1, \ldots, m$ such that for all $x=\left(x_{1}, \ldots, x_{m}\right)$ in $\mathbb{R}^{m}$

$$
\lim _{n \rightarrow+\infty} P\left(\frac{M_{n}-\mu_{n}}{\sigma_{n}} \leq x\right)=\lim _{n \rightarrow+\infty} F^{n}\left(\sigma_{n} x+\mu_{n}\right)=G(x)
$$

Furthermore, the $y_{i}$ are defined, for all $i=1, \ldots, m$ by the transformations

$$
y_{i}\left(x_{i}\right)=\left[1+\xi_{i}\left(\frac{x_{i}-\mu_{i}}{\sigma_{i}}\right)\right]_{+}^{-\frac{1}{\xi_{i}}} \text {, where } x_{+}=\max (x, 0),
$$

$\left\{\mu_{i} \in \mathbb{R}\right\},\left\{\xi_{i} \in \mathbb{R}\right\}$, and $\left\{\sigma_{i}>0\right\}$ being respectively location, shape, and scale parameters of the univariate margins $G_{i}$ of $G$. The generalized form of these margins $G_{i}$ is given by:

$$
G_{i}\left(x_{i}\right)=\left\{\begin{array}{l}
\exp \left\{-\left[1+\xi_{i}\left(\frac{x_{i}-\mu_{i}}{\sigma_{i}}\right)\right]_{+}^{-\frac{1}{\xi_{i}}}\right\} \text { if } \xi_{i} \neq 0, \\
\exp \left\{-\exp \left[-\left(\frac{x_{i}-\mu_{i}}{\sigma_{i}}\right)_{+}\right]\right\} \text {if } \xi_{i}=0 .
\end{array}\right.
$$

If (3.5) holds, $F$ is said to belong to the max-domain of attraction of $G$. This is equivalent to say that there exists an MEV distribution $G$ such as: $F\left(y_{1}, y_{2}, \ldots, y_{m}\right)=G_{*}\left(y_{1}, y_{2}, \ldots, y_{m}\right)$. 
For all $t \in S_{m-1} ; \mu$ being the angular measure on $S_{m-1}$ (see [8])

$$
G\left(y_{1}, y_{2}, \ldots, y_{m}\right)=\exp \left\{-\int_{S_{m}} \max \left(\frac{-q_{1}}{y_{1}}, \frac{-q_{2}}{y_{2}} ; \ldots, \frac{-q_{m}}{y_{m}}\right) \mu d(q)\right\}
$$

with $y_{i}>r_{i} ; i=1,2, \ldots, m$, where $r_{i}$ are thresholds. Then,

$$
F\left(x_{1}, \ldots, x_{m}\right)=\exp \left[-\int_{S_{m}} \max \left(-q_{1}\left(\frac{-1}{\log \left[F_{1}\left(x_{1}\right)\right]}\right)^{-1}, \ldots,-q_{m}\left(\frac{-1}{\log \left[F_{m}\left(x_{m}\right)\right]}\right)^{-1}\right) \mu d(q)\right],
$$

or

$$
F(x)=\exp \left[-\int_{S_{m}} \max \left(q_{1} \log \left[F_{1}\left(x_{1}\right)\right], \ldots, q_{m} \log \left[F_{i}\left(x_{m}\right)\right]\right) \mu d(q)\right] .
$$

Furthermore, suppose that $F_{i}$ is a GPD.

Then, $F_{i}\left(x_{i}\right)=1-\lambda_{i}\left[1+\xi_{i}\left(\frac{x_{i}-\mu_{i}}{\sigma_{i}}\right)\right]_{+}^{-\frac{1}{\xi_{i}}}$ if $t_{i}\left(x_{i}\right)=\left[1+\xi_{i}\left(\frac{x_{i}-\mu_{i}}{\sigma_{i}}\right)\right]_{+}^{-\frac{1}{\xi_{i}}}$.

Then it follows that: $\log \left(F_{i}\left(x_{i}\right)=\log \left(1-\lambda_{i} t_{i}\left(x_{i}\right)\right) \simeq \lambda_{i} t_{i}\left(x_{i}\right)\right.$.

Then, $F(x)=\exp \left[-\int_{S_{m}} \max \left(q_{1} \lambda_{1} t_{1}\left(x_{1}\right), \ldots, q_{m} \lambda_{m} t_{m}\left(x_{m}\right)\right) \mu d(q)\right]+o$ $\left(\max \left(\lambda_{i}\right)\right.$ if $B(x)=\int_{S_{m}} \max \left(q_{1} \lambda_{1} t_{1}\left(x_{1}\right), \ldots, q_{m} \lambda_{m} t_{m}\left(x_{m}\right)\right) \mu d(q)$, which is equivalent to

$$
B(x)=\lambda \sum_{i=1}^{i=m} t_{i}\left(x_{i}\right) \int_{S_{m}} \max \left(\frac{q_{1} t_{1}\left(x_{1}\right)}{\sum_{i=1}^{i=m} t_{i}\left(x_{i}\right)}, \ldots, q_{m}\left(1-\frac{\sum_{i=1}^{i=m-1} t_{i}\left(x_{i}\right)}{\sum_{i=1}^{i=m} t_{i}\left(x_{i}\right)}\right)\right) \mu d(q)
$$

where $\quad B(x)=\lambda \sum_{i=1}^{i=m} t_{i}\left(x_{i}\right) A\left(\tilde{t}_{1}\left(x_{1}\right), \ldots, \tilde{t}_{m-1}\left(x_{m-1}\right), \tilde{t}_{m}\left(x_{m}\right)\right) \quad$ or $\tilde{t}_{i}\left(x_{i}\right)=\frac{t_{i}\left(x_{i}\right)}{\sum_{i=1}^{i=m} t_{i}\left(x_{i}\right)}$. 
For the particular case of marginal distributions de Pareto standard parameter $\xi$ (i.e., $\mu_{i}=0 ; \sigma_{i}=1$ and $\xi_{i}=\xi, \xi>0 ; i=1,2, \ldots, m$ )

$$
B(x)=B_{m, \xi, \lambda}(x)=\lambda\left[m+\xi \sum_{i=1}^{i=m} x_{i}^{-\xi}\right] A\left(\widetilde{t}_{1}\left(x_{1}\right), \ldots, \widetilde{t}_{m-1}\left(x_{m-1}\right), \widetilde{t}_{m}\left(x_{m}\right)\right) .
$$

\subsection{Applications to usual some MEV distributions}

Using the relation (3.5), it is easy to establish that to propose the pseudo-dependence function for MEV consiste simply in giving its corresponding Pickands functions. So, we recall here some usual models of MEV distributions with the corresponding Pickands dependence function (see [2] and [9]).

\section{- Negative extension of logistic model of MEV distributions}

This model is given for all $x=\left(x_{1}, \ldots, x_{m}\right) \in \mathbb{R}^{m}$ and $\theta=\left(\theta_{1}, \ldots, \theta_{m}\right)$ by:

$$
G_{\theta}(x)=\exp \left\{-\sum_{i=1}^{m} x_{i}+\sum_{i<j}\left(x_{i}^{-\theta_{i j}}+x_{j}^{-\theta_{i j}}\right)^{\frac{-1}{\theta_{i j}}}+\sum_{k=3}^{m}(-1)^{k+1} \sum_{i=1}^{k} B_{1 \ldots k}\left(x_{i} x_{k}, \theta\right)\right\} .
$$

The corresponding Pickands function is given, for $t=\left(t_{1}, \ldots, t_{m-1}\right) \in S_{m}$ by

$$
\begin{aligned}
A_{\theta}(t)= & {\left[\sum_{i=1}^{m-1} t_{i}+\left(1-\sum_{i=1}^{m-1} t_{i}\right)+\sum_{i=1, i<j}^{m-2}\left(t_{i}^{-\theta_{i j}}+t_{j}^{-\theta_{i j}}\right)\right.} \\
& \left.+\sum_{i=1}^{m-1}\left(t_{i}^{-\theta_{i m}}+\left(1-\sum_{i=1}^{m-1} t_{i}\right)^{-\theta_{i m}}\right)^{\frac{-1}{\delta_{i m}}}\right]^{\frac{1}{\theta}}+R\left(t_{1}, \ldots, t_{m-1}, \delta_{12}, \ldots, \delta_{1 m}\right) ;
\end{aligned}
$$

where $R\left(t_{1}, \ldots, t_{m-1}, \delta_{12}, \ldots, \delta_{1 m}\right)$ is an integral rest. 


\section{- Logistic Gaussian model of MEV distribution}

This model is given for all $x=\left(x_{1}, \ldots, x_{m}\right) \in \mathbb{R}^{m}$ and $\theta=\left(\theta_{1}, \ldots, \theta_{m}\right)$ by:

$$
\begin{aligned}
G_{\theta}(x)= & \exp \left\{-\sum_{i=1}^{m} x_{i}+\sum_{1 \leq i<j \leq m}\left\{x_{i}+x_{j}-x_{i} \Phi\left(\frac{1}{\theta_{i j}}+\frac{\theta_{i j}}{2} \log \left(\frac{x_{i}}{x_{j}}\right)\right)\right.\right. \\
& \left.-x_{j} \Phi\left(\frac{1}{\theta_{i j}}+\frac{\theta_{i j}}{2} \log \left(\frac{x_{j}}{x_{i}}\right)\right)\right\} \\
& \left.+\sum_{S:|S| \geq 3}(-1)^{|S|+1} \int_{0}^{x_{i k}} \bar{\Phi}_{k-1}\left(\log \left(\frac{x}{x_{i}}\right)+\frac{2}{\delta_{i_{j}, i_{k}}^{2}} ; \Gamma\right) d x\right\}
\end{aligned}
$$

where $\bar{\Phi}_{k-1}(., \Gamma)$ is the survival function of the Gaussian model $(k-1)$-multivariate with variance-covariance matrix $\Gamma$ such as $\Gamma=\Gamma\left(\theta_{i_{j}, i_{k}}\right)$, a $(k-1)$-squared matrix where the elements $\left(i, j^{\prime}\right)$ are all equal to $2\left(\theta_{i_{j}, i_{k}}^{-2}+\theta_{i_{j^{\prime}}, i_{k}}^{-2}-\theta_{i_{j}, i_{j^{\prime}}}^{-2}\right)$ with $1 \leq j, j^{\prime} \leq k-1$ and $\theta_{i, i}^{-1}=0$ (see [7]).

The corresponding Pickands function is given, for $t=\left(t_{1}, \ldots, t_{m-1}\right) \in S_{m}$ by

$$
\begin{aligned}
A_{\theta}(t)= & 1-\sum_{i=1}^{m-1} t_{i}-\left(1-\sum_{i=1}^{m-1} t_{i}\right) \\
& +\sum_{i=1, i<j}^{m-2}\left(t_{i}+t_{j}-t_{i} \Phi\left(\frac{1}{\delta_{i j}}+\frac{\delta_{i j}}{2} \log \left(\frac{t_{i}}{t_{j}}\right)\right)-t_{j} \Phi\left(\frac{1}{\delta_{i j}}+\frac{\delta_{i j}}{2} \log \left(\frac{t_{j}}{t_{i}}\right)\right)\right)^{\frac{-1}{\delta_{i j}}} \\
& +\sum_{i=1}^{m-1}\left(t_{i}+t_{m}-t_{i} \Phi\left(\frac{1}{\delta_{i m}}+\frac{\delta_{i m}}{2} \log \left(\left(1-\sum_{k=1}^{m-1} t_{k}\right)^{-1}\right)\right)\right.
\end{aligned}
$$




$$
\begin{aligned}
& \left.-\left(1-\sum_{k=1}^{m-1} t_{k}\right) \Phi\left(\frac{1}{\delta_{i j}}+\frac{\delta_{i j}}{2} \log \left(\frac{1-\sum_{k=1}^{m-1} t_{k}}{t_{i}}\right)\right)\right) \\
& +R\left(t_{1}, \ldots, t_{m-1}, \delta_{12}, \ldots, \delta_{1 m}\right)
\end{aligned}
$$

where $R\left(t_{1}, \ldots, t_{m-1}, \delta_{12}, \ldots, \delta_{1 m}\right)$ is an integral rest.

\section{- Logistic mixed model of MEV distribution}

This model is given for all $x=\left(x_{1}, \ldots, x_{m}\right) \in \mathbb{R}^{m}$ and $\theta=\left(\theta_{1}, \ldots, \theta_{m}\right)$ by:

$$
G_{\theta}(x)=\exp \left\{-\left[\sum_{1 \leq i<j \leq m}\left(\left(p_{i} x_{i}^{\theta}\right)^{\theta_{i j}}+\left(p_{j} x_{j}^{\theta}\right)^{\theta_{i j}}\right)^{\frac{1}{\theta_{i j}}}+\sum_{i=1}^{m} \nu_{i} p_{i} x_{i}^{\theta}\right]^{\frac{1}{\theta}}\right\} .
$$

The corresponding Pickands function is given, for $t=\left(t_{1}, \ldots, t_{m-1}\right) \in S_{m}$ by

$$
\begin{aligned}
A_{\theta}(t)= & {\left[\sum_{1 \leq i<j \leq m-1}\left(\left(p_{i} t_{i}^{\theta}\right)^{\theta_{i j}}+\left(p_{j} t_{j}^{\theta}\right)^{\theta_{i j}}\right)^{\frac{1}{\theta_{i j}}}+\sum_{i=1}^{m} \nu_{i} p_{i} t_{i}^{\theta}\right.} \\
& \left.\sum_{1 \leq i \leq m-1}\left(\left(p_{i} t_{i}^{\theta}\right)^{\theta_{i j}}+\left(p_{j}\left(1-\sum_{1 \leq k \leq m-1} t_{k}\right)^{\theta}\right)^{\theta_{i j}}\right)^{\frac{1}{\theta_{i j}}}+\sum_{i=1}^{m} \nu_{i} p_{i} t_{i}^{\theta}\right]^{\frac{1}{\theta}} .
\end{aligned}
$$




\section{- Logistic asymmetric model of MEV distribution}

This model is given for all $x=\left(x_{1}, \ldots, x_{m}\right) \in \mathbb{R}^{m}$ and $\theta=\left(\theta_{1}, \ldots, \theta_{m}\right)$ by:

$$
\begin{aligned}
G_{\theta}\left(y_{t}^{\breve{s}}\right)= & \exp \left\{-\sum_{k=1}^{m-1} y_{t}^{(i)}+\sum_{S:|S| \geq 1}^{m}(-1)^{|S|+1}\left[\sum_{i \in S}^{m-1} y_{t}^{(k) \theta}\right.\right. \\
& \left.\left.-\sum_{i<j ; i, j \in S}\left[p^{-\theta_{i j}} y_{t}^{(i)}+p_{j}^{-\delta_{i j}} x_{j}^{\theta \theta_{I j}}\right]^{\frac{-1}{\theta_{i j}}}\right]^{\frac{-1}{\theta}}\right\} .
\end{aligned}
$$

The corresponding Pickands function is given, for $t=\left(t_{1}, \ldots, t_{m-1}\right) \in S_{m}$ by

$$
\begin{aligned}
A_{\theta}(t)= & -\sum_{S: 2 \leq|S| \leq m-1}(-1)^{|S|+1}\left[\sum_{i \in S} t_{i}^{-\theta}+-\sum_{i<j ; i, j \in S}\left[p_{i}^{-\theta_{i j}} t_{i}^{\theta \theta}+p_{j}^{-\delta_{i j}} t_{j}^{\theta \theta_{I j}}\right]^{\frac{-1}{\theta_{i j}}}\right] \\
& +(-1)^{m+1}\left[\left(1-\sum_{1 \leq i \leq m-1} t_{i}\right)^{\theta}\right. \\
& \left.-\sum_{i<m}\left[p_{i}^{-\theta_{i m}} t_{i}^{\theta \theta_{i m}}+p_{m}^{-\theta_{i j}}\left(1-\sum_{1 \leq i \leq m-1} t_{i}\right)^{\theta \theta_{i m}}\right]^{\frac{-1}{\theta_{i m}}}\right] .
\end{aligned}
$$

\section{Conclusion}

The results of the study provides important characterizations of parametric max-stable processes. Especially, they show that the concept of Archimax copulas can also be extended to three-dimensional case. Moreover, multivariate generalized Pareto distributions are characterized by a pseudo-dependence function while usual multivariate extremal models are given with the corresponding dependence function. 


\section{References}

[1] Barro Diakarya, Diallo Moumouni and Bagré Remi Guillaume, Spatial tail dependence and survival stability in a class of Archimedean copulas, Inter. J. Math. Math. Sci. 2016 (2016), Article ID 8927248, 8 pp.

http://dx.doi.org/10.1155/2016/8927248

[2] Barro Diakarya, Conditional dependence of trivariate generalized Pareto distributions, Asian Journal of Mathematics \& Statistics 2(2) (2009), 20-32.

DOI: 10.3923/ajms.2009.20.32

[3] J. Beirlant, Y. Goegebeur, J. Segers and J. Teugels, Statistics of Extremes: Theory and Application-Wiley, Chichester, England, 2005.

[4] A. Charpentier and J. Segers, Tails of multivariate Archimedean copulas, J. Multivariate Anal. 100(7) (2009), 1521-1537.

http://dx.doi.org/10.1016/j.jmva.2008.12.015

[5] M. Degen, On Multivariate Generalised Pareto Distributions and High Risk Scenarios - Thesis, Department of Mathematics, ETH Zürich, 2006.

[6] C. Genest and J. Mackay, Copules archimédiennes et familles de lois bidimensionnelles dont les marges sont données, Canadian Journal of Statistics 14 (1993), 145-159.

[7] J. Husler and R.-D. Reiss, Extreme Value Theory, Proceedings of a Conference held in Oberwolfach, Dec. 6-12, 1987. Springer, Berlin etc. H.-J. Lenz, 1989.

[8] H. Joe, Multivariate Models and Dependence Concepts, Monographs on Statistics and Applied Probability 73, Chapman and Hall, London, ISBN 978-0-412-7331 (1997).

[9] S. I. Resnick, Extreme Values, Regular Variation and Point Processes-SpringerVerlag, 1987.

[10] N. Tajvidi, Characterisation and Some Statistical Aspects of Univariate and Multivariate Generalised Pareto Distributions, Department of Mathematics, Chalmers Tekniska Hogskola Goteborg, 1996.

http://www.math.chalmers.se/ nader/thesis.ps 